\title{
Unveiling Nanometric Plasmons Optical Properties With Advanced Electron Spectroscopy in the Scanning Transmission Electron Microscope
}

M Kociak ${ }^{1}$, A. Losquin ${ }^{1}$, N. Kawasaki ${ }^{2}$, H. Lourenço-Martins ${ }^{1}$, A. Campos ${ }^{1}$, P. Das ${ }^{1}$, S. Meuret ${ }^{1}$, M. Tencé $^{1}$, L F Zagonel ${ }^{3}$, K. March ${ }^{1}$, F. J. Javier Garcia de Abajo ${ }^{4}$, O. Stéphan ${ }^{1}$

1. Laboratoire de Physique des Solides, CNRS UMR8502, Université Paris-Sud XI, Orsay, France

2. Morphological Research Laboratory, Toray Research Center Inc., Otsu, Shiga Japan

3. Gleb Wataghin" Institute of Physics - University of Campinas - UNICAMP - 13083-970, Campinas, São Paulo, Brazil

4. ICFO-Institut de Ciencies Fotoniques, Mediterranean Technology Park, Castelldefels (Barcelona), Spain

Since the pioneering work of Yamamoto and co-workers [1], the use of electron spectroscopy such as Cathodoluminescence (CL) and Electron Energy Loss Spectroscopy (EELS) in a Scanning (Transmission) Electron Microscope (STEM) has considerably helped improving our understanding of the optical properties of metallic nanoparticles. The resemblance of spectroscopic signals between electron and optical techniques leads to the intuition that both types of excitations (optical and electronic) give very similar information, an idea theoretically discussed quite early $[2,3]$.

However, it is also quite intuitive that CL and EELS should be different [2,4]. For example, EELS helps at detecting any sort of modes while CL can only detect radiative ones. On the other hand, even between different optical spectroscopy techniques, such as the measurement of extinction or scattering crosssections, clear differences are expected when studying plasmons, like energy shifts or spectral shape changes. The lack of adapted instrumentation capable of performing combined EELS and CL, as well as theoretical developments allowing to account for the generic difference between EELS and CL and their optical counterparts impeached a comprehensive understanding of plasmons physics with the otherwise amazing electron spectroscopy techniques.

In this talk, we will present recent experimental results showing combined EELS and CL spectral mapping in the STEM of plasmonic properties for nanoparticles with various shapes (triangles [3], cubes ...) and composition (gold [5], silver [6], aluminum...). The instrumentation necessary to reach a relevant level of accuracy in the comparison between both techniques will be discussed. In particular, the question of wavelength and intensity calibration will be evoked.

Helped with different theoretical tools [3,7], we will try to show how these results can be related to their optical counterparts (extinction, scattering), and what type of physical insights can be gained from these combined measurements. Finally, if time allows, pointing the weaknesses of state-of-the-art CL and EELS (in terms of spectral range and/or spectral resolution), we will present EELS results obtained on highly monochromated electron beams that could cope with these limitations [8]. 
References:

[1] N. Yamamoto et al. Phys. Rev. B 64, (2001).

[2] F. Garcia de Abajo, Phys. Rev. B 59, 3095 (1999).

[3] F. García de Abajo and M. Kociak, Phys. Rev. Lett. 100, (2008).

[4] M. Kociak and O. Stéphan, Chem. Soc. Rev. 43, 3865 (2014).

[5] A. Losquin et al., Nano Lett. 15, 1229 (2015).

[6] N. Kawasaki, et al. In Preparation (2016).

[7] A. Losquin and M. Kociak, ACS Photonics 2, 1619 (2015).

[8] K. March et al., In Preparation (2016).

[9] M. Kociak et al., Comptes Rendus Physique 15, 158 (2014).
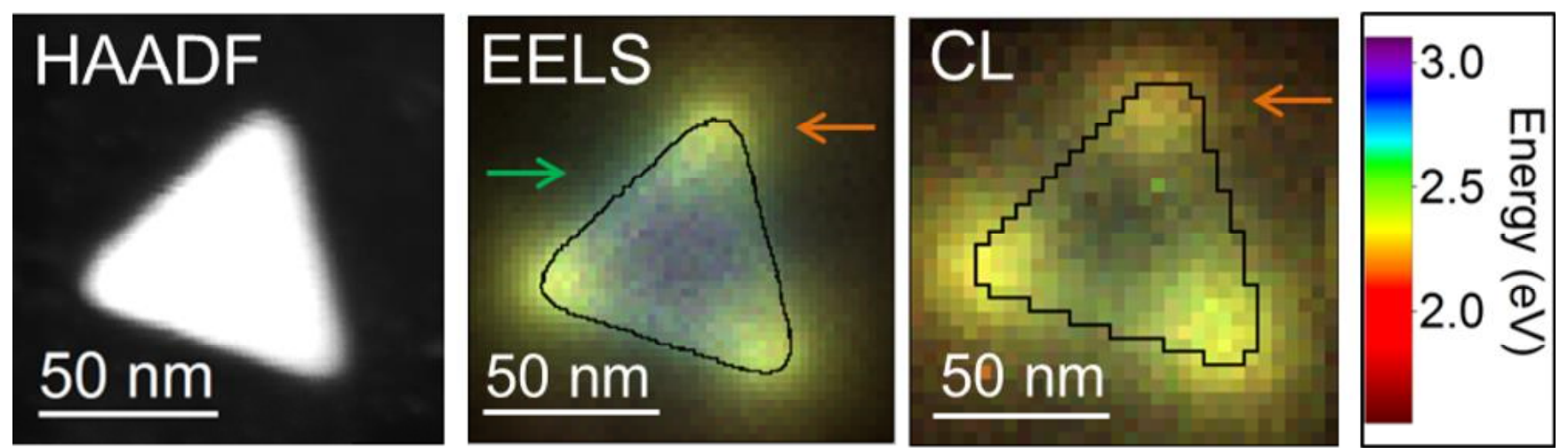

Figure 1. Combined EELS and CL spectral-imaging of a gold nanoprism (after [5]). Left: HAADF image of the nanoprism. Center: Synthetic representation [9] of an EELS spectral-image. To create such color image, each energy layer of the spectral image is given a different color, the energy-color correspondence being given on the color scale on the right. Two modes show up in this representation, one at low energy localized on the tip, and one at higher energy on the edge. Right: Similar representation for a CL spectral-image acquired on the same nanoprism. Only the low energy mode shows up, as a strong indication that the low energy mode is of dipolar type and that the prism is too small to sustain radiative modes of higher order. 